\title{
Measured Temporal and Spectral PMD Characteristics and Their Implications for Network-Level Mitigation Approaches
}

\author{
Christopher T. Allen, Senior Member, IEEE, Pradeep Kumar Kondamuri, Douglas L. Richards, and Douglas C. Hague
}

\begin{abstract}
Signal degradation due to polarization-mode dispersion (PMD) effects may become significant for signaling rates of $10 \mathrm{~Gb} / \mathrm{s}, 40 \mathrm{~Gb} / \mathrm{s}$, and beyond. To assess the utility of various PMD mitigation schemes, temporal and spectral measurements of differential group delay (DGD) were made on $95 \mathrm{~km}$ of buried standard single-mode fiber over an 86-d period to determine the distribution and rate of change of high-DGD events. As expected, statistical analysis of variations in DGD indicate that excursions from the mean DGD by factors of 3.7 or higher have very low probability. For this link, the DGD varied slowly with time (having a drift time of about $3.4 \mathrm{~d}$ ) and rapidly with wavelength. The DGD data agree well with results of similar experiments reported in the literature. Statistical analysis of the measured DGD data shows that high-DGD episodes will be exceedingly rare and short-lived. The impact of PMD on network operations is explored and approaches to ensure network reliability are reviewed for network operators given the task of transporting high-bit-rate channels over fiber links with known PMD characteristics.
\end{abstract}

Index Terms-Optical fiber characterization, optical fiber communication systems, polarization drift, polarization-mode dispersion (PMD), polarization-mode dispersion outage.

\section{INTRODUCTION}

$\mathbf{I}$ $\mathrm{N}$ THE PHENOMENON called polarization-mode dispersion (PMD), birefringence in the optical fiber provides two polarization-dependent group velocities for optical signals. In the high-coherence model of PMD (which assumes that the coherence time of the light source is greater than the PMD-induced delays and no polarization-dependent loss), an input pulse will result in two orthogonally polarized pulses that preserve the shape of the original input pulse. The relative amplitudes of these two pulses is determined by the state of polarization (SOP) of the input pulse relative to the fiber's input principal states of polarization (PSPs). Thus, for each pulse input, two pulses arrive at the receiver with different arrival times, called the differential group delay (DGD) $\Delta \tau$. This first-order model is frequency-independent and is only valid over limited bandwidths. For wider bandwidths, higher order effects must be considered, resulting in frequency-dependent polarization-mode dispersion [1], [2]. The bandwidth over which the PSPs can be assumed constant

Manuscript received April 15, 2002; revised July 30, 2002. This work was supported by Sprint Corporations Co., L.P.

C. T. Allen and P. K. Kondamuri are with the Lightwave Communication Systems Laboratory, Information and Telecommunications Technology Center (ITTC), University of Kansas, Lawrence, KS 66045 USA (e-mai callen@eecs.ukans.edu).

D. L. Richards and D. C. Hague are with the Sprint Corporation, Overland Park, KS 66251 USA.

Digital Object Identifier 10.1109/JLT.2003.808634 depends on the properties of the fiber and has been shown to vary inversely with the mean DGD, $\langle\Delta \tau\rangle$ [3]. While the minimum bandwidth of the PSPs in single-mode fibers (SMFs) was found to be always over $50 \mathrm{GHz}$ [3], this bandwidth for standard SMF is of the order of $100 \mathrm{GHz}$ [1].

PMD may become a major impediment for network operators seeking to increase the per-channel data rate on long-haul fiber-optic links. While the DGD in buried fiber had negligible impact at $2.5-\mathrm{Gb} / \mathrm{s}$ signaling rates, upgrades to $10 \mathrm{~Gb} / \mathrm{s}, 40$ $\mathrm{Gb} / \mathrm{s}$, and beyond will require increasingly more attention. While there are PMD challenges facing carriers at $10 \mathrm{~Gb} / \mathrm{s}$, these challenges are not as severe as originally feared. Major carriers are successfully deploying $10-\mathrm{Gb} / \mathrm{s}$ dense-wavelength-division-multiplexed (DWDM) links across the core of their networks. A marked improvement in the DGD tolerance of $10-\mathrm{Gb} / \mathrm{s}$ long-reach receivers (to about $40 \mathrm{ps}$ ) will likely satisfy most length demands, obviating the need for PMD compensation (PMDC). Signaling rates of $40 \mathrm{~Gb} / \mathrm{s}$ and beyond will most likely require some form of mitigation in long-haul applications, such as robust modulation schemes or PMDC.

To ensure signal quality on their fiber at higher bit rates, network engineers must anticipate the impact of PMD on the various fiber routes. Design of a reliable network requires a good model of the PMD characteristics on each link. An understanding of the variability of both the DGD and the PSPs is required to specify appropriate transmission parameters as well as PMDC specifications. Factors such as the mean DGD, PMD correlation time, and bandwidth, as well as second-order effects, together with performance prediction models, can provide this understanding. While the probabilistic properties of PMD variations are known, the characteristics of a particular link depend on how it was cabled and installed. Therefore, PMD measurements on installed fiber links are required.

While PMD is a vector quantity, with a magnitude (DGD) and a direction (PSP), we are deliberately focusing exclusively on DGD since this is a readily measured parameter on installed optical networks. The statistical distribution and behavior of PSPs has been extensively studied and reported elsewhere.

\section{PMD STATISTICS}

\section{A. Mean DGD}

For long optical fibers, the PMD figure of merit typically specified is its mean DGD $\langle\Delta \tau\rangle$ (having units of ps) or its PMD coefficient $\langle\Delta \tau\rangle / \sqrt{ } L$ (having units of ps/ $\sqrt{ } \mathrm{km}$ ), where $L$ is the fiber length. The PMD for an installed (buried) fiber-optic cable 
is dominated by the inherent PMD of the bare fiber; however, the level of relaxation provided by the cabling and installation techniques also affects PMD. While the PMD in bare fiber is determined largely by the core-cladding concentricity achieved during manufacture, we have found that loose-tube cabling results in a lower PMD than other cabling methods, such as slotted core cabling. In addition, mechanical stresses introduced during cable installation (burial) also contribute to the PMD and will be affected by the installation practices used and whether the cable is in a protective conduit.

The mean DGD for a given fiber is a constant that represents both the average of DGD values at one time across a broad spectral bandwidth

$$
\langle\Delta \tau\rangle_{\lambda}=\frac{1}{N_{\lambda}} \sum_{i=1}^{N_{\lambda}} \Delta \tau\left(\lambda_{i}, t\right)
$$

and the average of DGD values for a single wavelength over a long time period

$$
\langle\Delta \tau\rangle_{t}=\frac{1}{N_{t}} \sum_{i=1}^{N_{t}} \Delta \tau\left(\lambda, t_{i}\right)
$$

where $\Delta \tau(\lambda, t)$ is the DGD value at wavelength $\lambda$ and time $t$. Although the mean DGD for an installed fiber is constant, changing environmental factors (e.g., temperature) cause the instantaneous DGD at a given wavelength $\Delta \tau(\lambda, t)$ to vary randomly about that mean.

\section{B. Maxwellian Distribution}

The DGD for a given wavelength at any moment in time $\Delta \tau(\lambda, t)$ is a random variable with a Maxwellian probability density function (pdf) [4], [5]

$$
p(\Delta \tau)=\sqrt{\frac{2}{\pi}} \frac{\Delta \tau^{2}}{\sigma^{3}} e^{\left(-\Delta \tau^{2} / 2 \sigma^{2}\right)}, \text { where } \sigma=\langle\Delta \tau\rangle \sqrt{\frac{\pi}{8}}
$$

for $0<\Delta \tau<+\infty$. Therefore, the single parameter $\langle\Delta \tau\rangle$ fully specifies the distribution.

Using this distribution, the probability of $\Delta \tau$ exceeding a particular value can be found using

$$
P(\Delta \tau \geq X)=1-\int_{0}^{X} p(\Delta \tau) d \Delta \tau .
$$

For example, the probability of $\Delta \tau /\langle\Delta \tau\rangle$ exceeding 3.7 is $1.3 \times 10^{-7}$. Expressed another way, if the mean DGD of a fiber link is $10 \mathrm{ps}$, then $99.99999 \%$ of the time, the DGD will be less than $37 \mathrm{ps}$.

\section{Network Design CONSIDERATIONS}

In the design of a robust, long-haul fiber-optic network, the relationship between the maximum achievable link length and bit rate must be considered. For link designs where the maximum tolerable DGD is exceeded, techniques for coping with the effects of PMD must be explored.

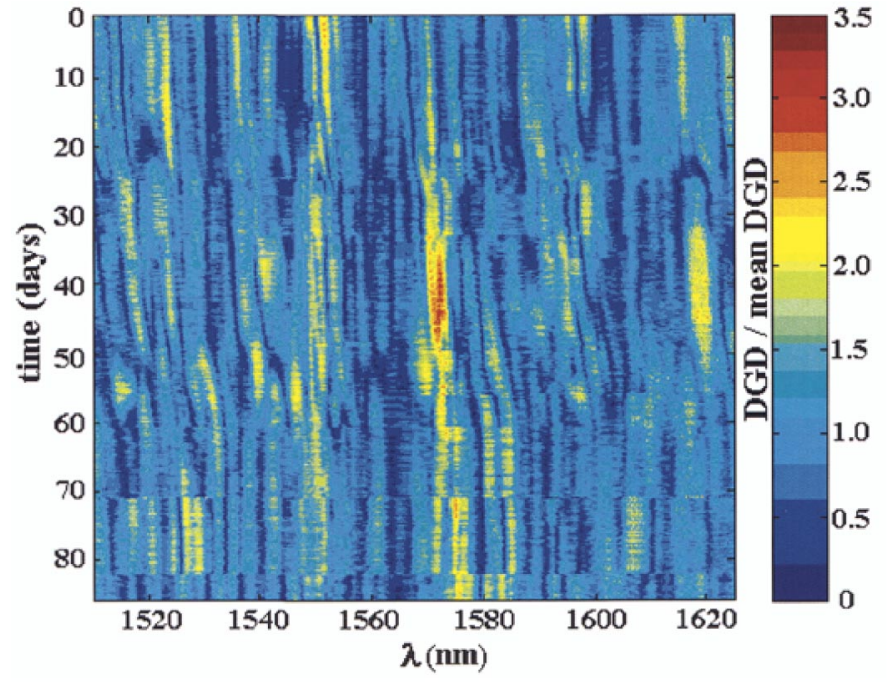

Fig. 1. Map of normalized DGD versus wavelength and time.

\section{A. Receiver DGD Tolerance}

The maximum-link DGD that a receiver can tolerate before the signal degradation becomes unacceptable depends on a variety of factors, including line bit rate, modulation format, optical signal-to-noise ratio (SNR) and receiver design. For intensity-modulated, direct-detected (IM-DD) systems, Iannone et al. [6] found that when the transmitted signal excites both PSPs equally (a worst-case condition), a 1-dB receiver sensitivity penalty results when the instantaneous DGD is about $23 \%$ of the signaling time period $T_{\mathrm{bit}}$. For a $2.5-\mathrm{Gb} / \mathrm{s}$ nonreturn-to-zero (NRZ) signal ( $T_{\mathrm{bit}}$ is $400 \mathrm{ps}$ ), this corresponds to a tolerable DGD value of about $92 \mathrm{ps}$; at $10 \mathrm{~Gb} / \mathrm{s}$, about 23 ps is tolerable; and for a $40-\mathrm{Gb} / \mathrm{s} \mathrm{NRZ} \mathrm{signal,} \mathrm{this}$ corresponds to about $5.7 \mathrm{ps}$. This maximum-tolerable DGD level is representative of the NRZ IM-DD case; receiver DGD tolerance can be improved through careful receiver design, the use of PMD-tolerant signaling formats, and the use of forward-correction codes (FECs). Khosravani and Willner [7] showed that return-to-zero (RZ), chirped RZ, and dispersion-managed soliton signaling formats are much more tolerant of PMD effects, compared with NRZ formats. Shieh et al. [8] and Xie et al. [9] have demonstrated a substantial increase in receiver tolerance of DGD when the FEC is used. Modern long-haul, 10-Gb/s receivers using FEC or RZ modulation can tolerate about $40 \mathrm{ps}$ of DGD with a 1-dB power penalty.

\section{B. Probability of Signal Outage}

For occurrences of high instantaneous DGD, signal quality may be intolerable, resulting in a PMD-induced outage. Such outages may significantly affect network availability for higher bit rates $(10 \mathrm{~Gb} / \mathrm{s}, 40 \mathrm{~Gb} / \mathrm{s}$, and higher). For a network to operate with an overall availability of "five nines" (i.e., $99.999 \%$ of availability), the desired PMD-related availability factor may be "seven nines" (i.e., 99.999 99\%), which corresponds to a maximum-tolerable DGD 3.7 times the mean DGD. For a $2.5-\mathrm{Gb} / \mathrm{s}$ IM-DD NRZ system with a DGD tolerance of $92 \mathrm{ps}$, this results in an acceptable mean DGD value of $25 \mathrm{ps}$; for a $10-\mathrm{Gb} / \mathrm{s}$ system with a DGD tolerance of $23 \mathrm{ps}$, the acceptable mean DGD is 6.2 




Fig. 2. Histogram of measured DGD/ mean DGD data, along with Maxwellian pdf for comparison.

ps; and for 40-Gb/s with a tolerable DGD of $5.7 \mathrm{ps,} \mathrm{the} \mathrm{accept-}$ able mean DGD level is 1.5 ps. For DGD-tolerant receivers (40 ps at $10 \mathrm{~Gb} / \mathrm{s})$, this results in an acceptable-mean DGD of 10.8 ps.

\section{Coping With PMD}

For network operators faced with the challenge of upgrading the channel data rate on a high-PMD link in the network, a handful of solutions exist that will preserve the signal quality at increased data rates.

One alternative cost solution is to selectively replace those fiber segments in the link known to be the dominant contributors to the overall link DGD, if they can be identified. Another alternative cost solution is to regenerate the optical signal by placing back-to-back terminals at the point in the link where the DGD effects approach an intolerable level, thus effectively reducing the optical link length.

Still another approach is to introduce error-correction codes, such as FEC. In this approach, the optical data payload is reduced incrementally in exchange for a marginal gain in PMD tolerance.Yet another solution is to incorporate an adaptive PMDC system [8]-[12], typically located at the receiver. Typical PMD compensation systems are effective at minimizing the effects of first-order PMD and, in some cases, second-order PMD. However, both first- and second-order PMDC systems suffer the drawback that they reduce the effects of signal degradation over a very narrow optical bandwidth. This is a significant drawback for DWDM systems. For a long-haul fiber-optic link carrying hundreds of wavelengths, a separate PMDC system may be required for each wavelength to provide the desired seven-nines availability.

For DWDM systems, another potential solution exists. Särkimukka et al. [13] proposed a method for mitigating PMD effects in a multichannel system by moving traffic off of PMD-impaired channels onto spare channels that are not experiencing PMD degradation.

One may also rely upon more traditional protection techniques, e.g., SONET ring or Internet protocol (IP) routing at layers 1 and 3, respectively. This protection can easily provide a guard against occasional PMD-induced outages of limited duration. However, for this approach to be viable, the episodes of abnormally high-DGD events must be infrequent and spectrally localized. To evaluate the feasibility and limits of this solution, an understanding of the temporal and spectral nature of PMD is required.

Finally, there are also efficient optical networking solutions offering varying degrees of protection by using an optical cross connect with a DWDM system. Operators may then construct a mesh-protected network and provide managed wavelength services that are protected against possible PMD-induced outages. Similar to the traditional protection methods, these more recent techniques will only be viable with infrequent and spectrally localized outages.

\section{TEMPORAL BEHAVIOR OF DGD}

Given the dynamic nature of PMD and the low probability of excursions to intolerable levels, measurements of $\Delta \tau(\lambda, t)$ on buried fiber spans were made over long periods to enable prediction of the potential impact of PMD on network availability. Of particular interest are the frequency and duration of these rare events. The Jones matrix eigenanalysis (JME) technique was used to measure the DGD data on a $95-\mathrm{km}$ span of slotted-core, direct buried, standard single-mode (ITU G.652) fiber-optic cable made available by Sprint.

DGD was measured roughly every $3 \mathrm{~h}$ at wavelengths ranging from $1510 \mathrm{~nm}$ to $1625 \mathrm{~nm}$ with a spectral resolution of $0.1 \mathrm{~nm}$ (about $12.5 \mathrm{GHz}$ ). Over $86 \mathrm{~d}$ (from November 9, 2001, through February 2, 2002) 692 measurements were made on the 1150 

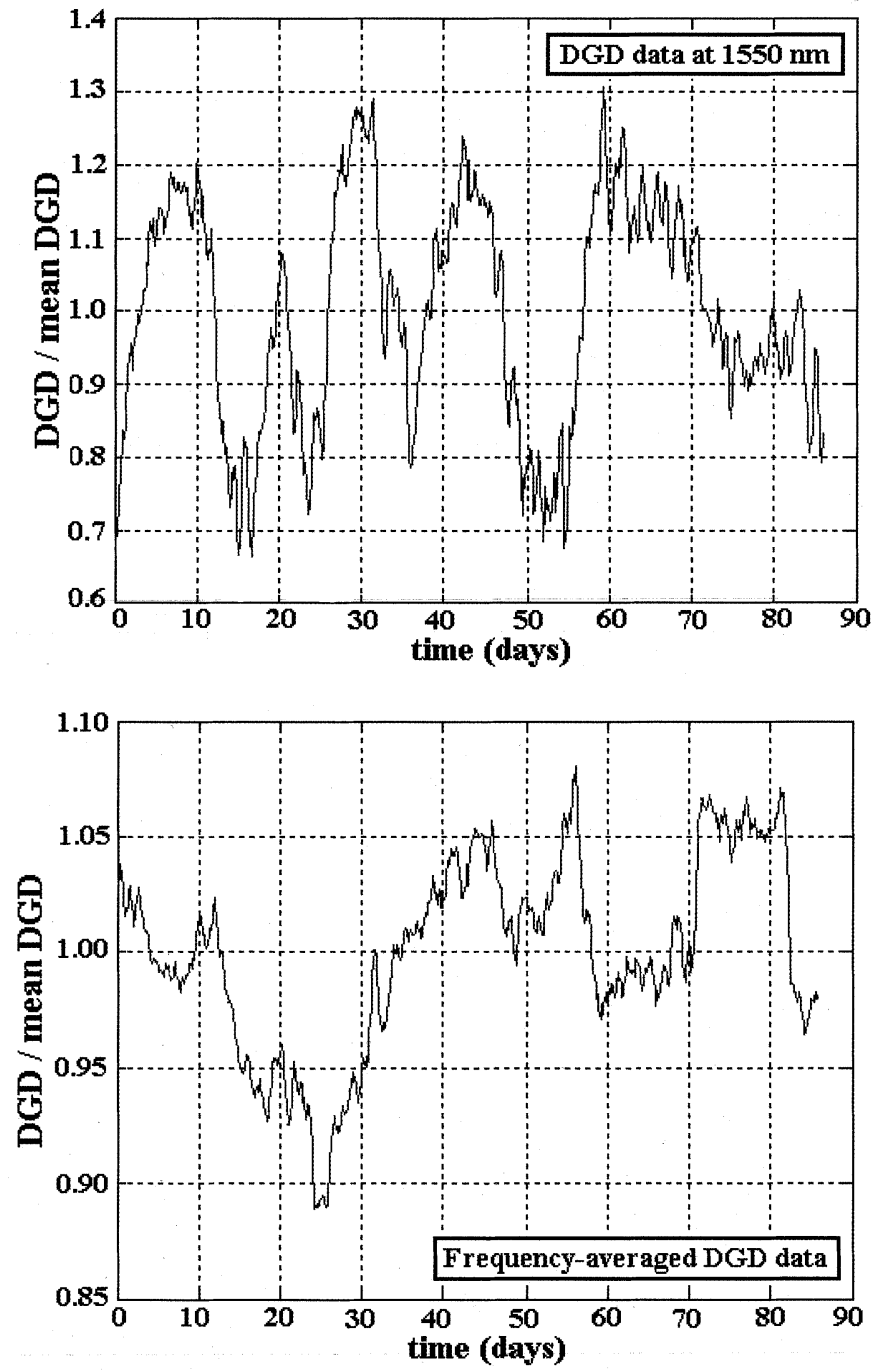

Fig. 3. Measured temporal variations in normalized DGD over $86 \mathrm{~d}$ at 1550 $\mathrm{nm}$ (top) and averaged overall 1150 frequency measurements (bottom).

discrete wavelengths. Fig. 1 shows in a color-coded format this normalized DGD data (i.e., $\Delta \tau /\langle\Delta \tau\rangle)$ representing 795800 measured values.

A histogram of this normalized DGD data is shown in Fig. 2 and is seen to have a shape consistent with a Maxwellian distribution, as expected. A curve representing a Maxwellian distribution for a 1-ps mean DGD is superimposed for comparison. Note that no occurances of DGD/mean DGD greater than 3.1 were observed during this $86-\mathrm{d}$ period.

From Fig. 1, it is apparent that for buried fiber, DGD values do not change rapidly (i.e., no abrupt changes are seen). Fig. 3 shows time histories of measured DGD data over the 86-d period. The top plot is DGD data at $1550 \mathrm{~nm}$, and the bottom plot is frequency-averaged data. While the mean value of the bottom plot is one (by definition), the mean value of the top plot is 1.088 . This should not be interpreted to mean that the mean DGD is changing; rather, since fewer data were used to estimate the mean, there is more uncertainty in that estimate, compared with the estimate using all of the data.

To determine the DGD rate of change, an autocorrelation analysis was performed on the DGD time histories. Fig. 4 (top)
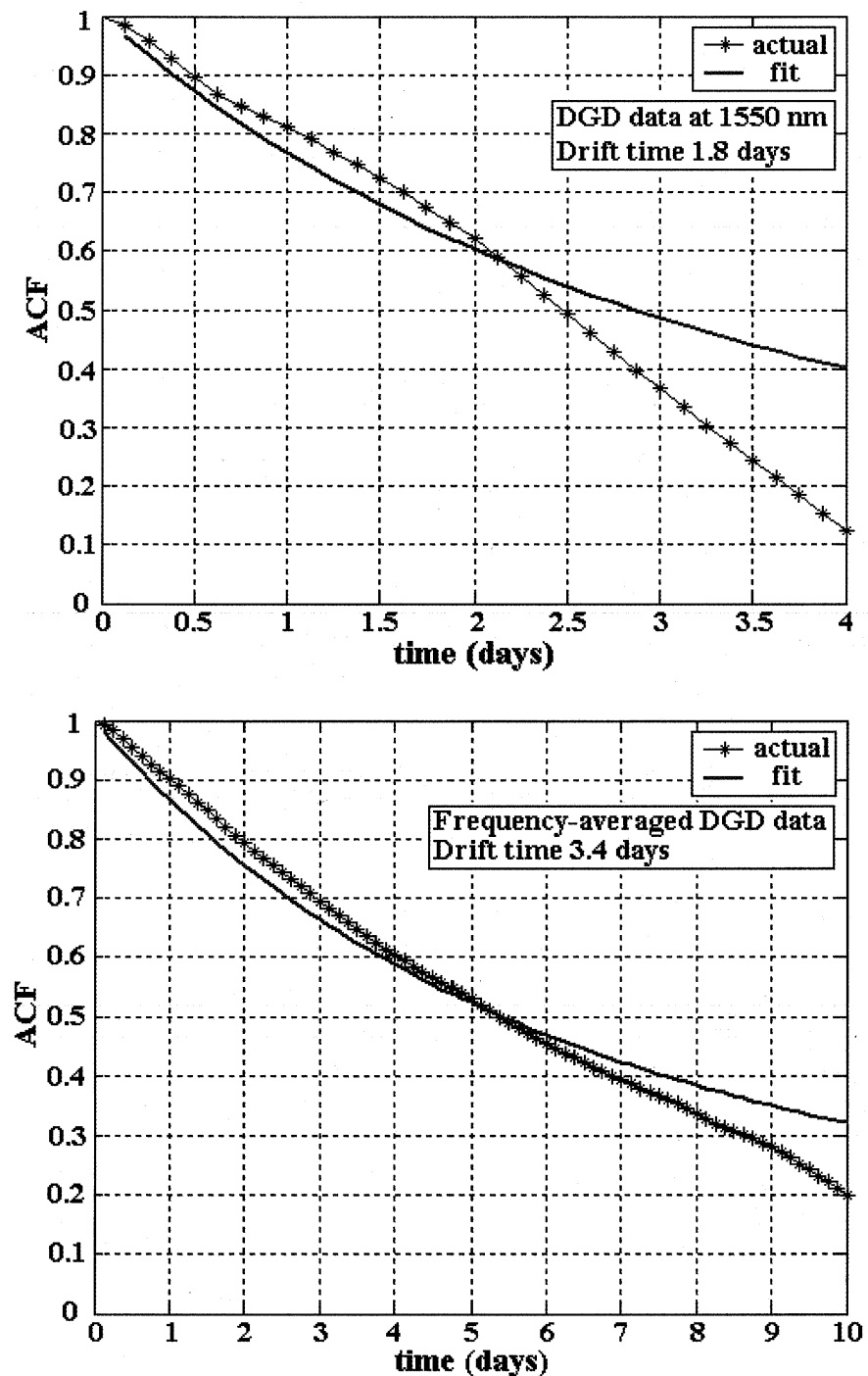

Fig. 4. Normalized temporal ACFs of normalized DGD data measured at 1550 nm (top) and across 1150 frequencies (bottom). Theoretical ACF curves are fitted to the measured temporal ACFs.

shows the normalized temporal autocorrelation function (ACF) of the DGD data measured at $1550 \mathrm{~nm}$. Fig. 4 (bottom) shows the ACF for the DGD time history for frequency-averaged DGD data. Also shown in Fig. 4 are curves representing the theoretical temporal ACF for DGD [14], which has the form

$$
\operatorname{AFC}(\Delta t)=\frac{1-\exp \left(\frac{-|\Delta t|}{t_{d}}\right)}{\frac{|\Delta t|}{t_{d}}}
$$

where $t_{d}$ is the average drift time of DGD. The drift time indicates the time scale over which the DGD changes. Furthermore, when outages occur, the outage duration will be related to the drift time [14], [15]. Based on data collected over $86 \mathrm{~d}$, the drift time for this fiber is estimated to be around 3.4 d. Expressed another way, samples should be collected about once every $3 \mathrm{~d}$ to obtain statistically independent DGD values on a specific wavelength; measurements collected more often are correlated.

For comparison, others have reported a range of DGD correlation times under various fiber conditions. For spools of fiber 

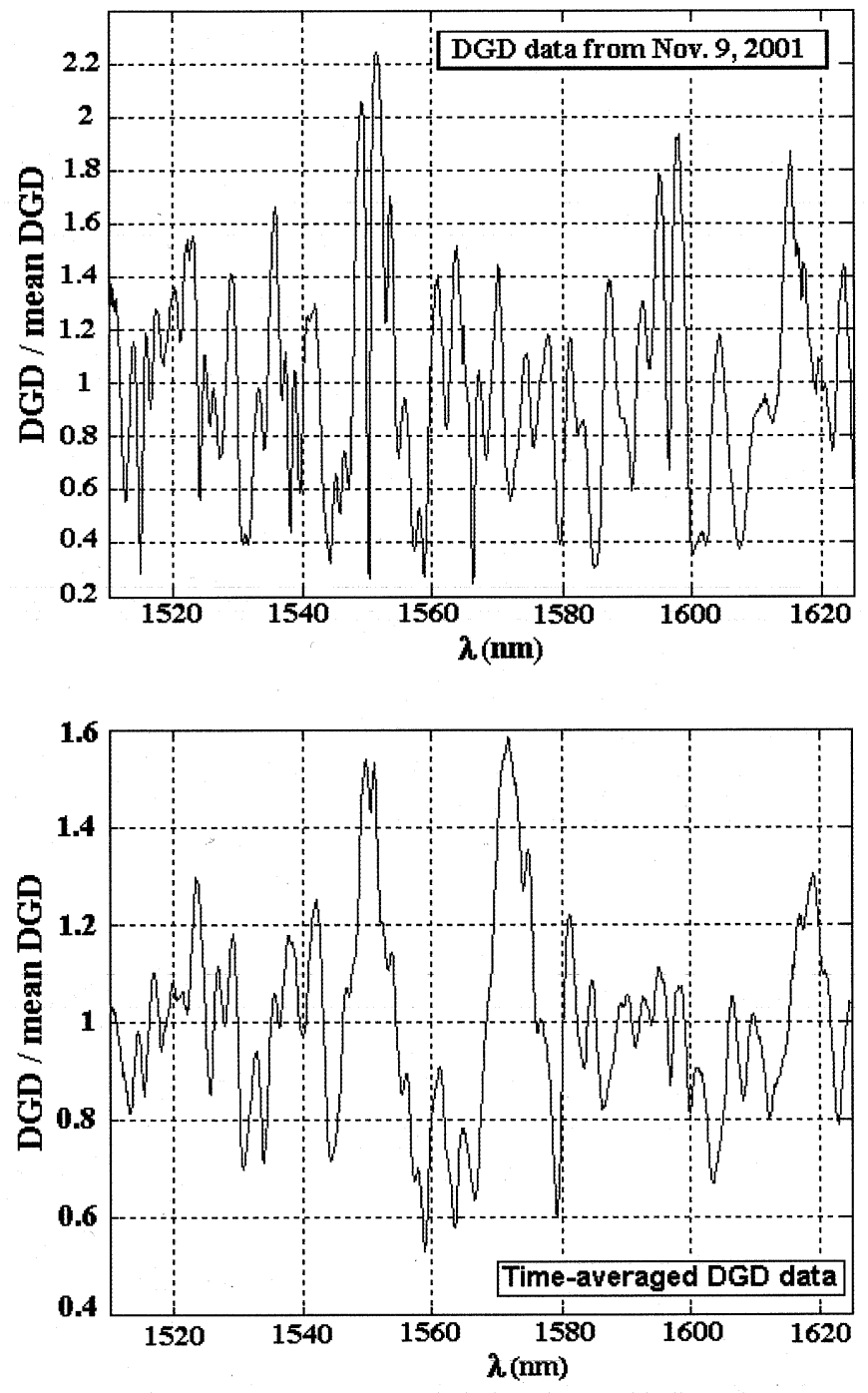

Fig. 5. Spectral variations in normalized DGD over 1150 wavelengths measured on November 9, 2001 (top), and time-averaged overall 692 time measurements (bottom).

in a laboratory environment, correlation times of about $30 \mathrm{~min}$ on $31.6 \mathrm{~km}$ of fiber [16] and $3 \mathrm{~h}$ on a 10-km fiber [17] have been reported. DGD variations on a $48-\mathrm{km}$ aerial cable exhibited time scales ranging from 5 to $90 \mathrm{~min}$, depending the air temperature rate of change [18]. For submarine cables, a DGD correlation time of about $1 \mathrm{~h}$ was observed on a 119-km cable [19], and [20] observed PMD changes with a period of about 2 mo on a 62-km fiber-optic cable. On buried fibers, correlation times of at least $20 \mathrm{~min}(17 \mathrm{~km})$ [21], 1-2 $\mathrm{h}(48.8 \mathrm{~km})$ [18], 3 and 5.7 $\mathrm{d}(127 \mathrm{~km})$ [14], and $19 \mathrm{~h}(114 \mathrm{~km})$ [22] have been reported. The significant variation of correlation times demonstrates how the installation scheme impacts the temporal behavior of DGD. Since temperature variations are known to cause PMD variations, cables in a thermally stable environment (e.g., submarine cable) will have long correlation times, whereas cables that experience diurnal temperature variations (e.g., aerial cables and buried cables with above-ground segments) will have correlation times less than $24 \mathrm{~h}$. And cables in an unstable thermal and mechanical environment (e.g., aerial cables) will have correlation times dependent on both temperature and wind conditions.
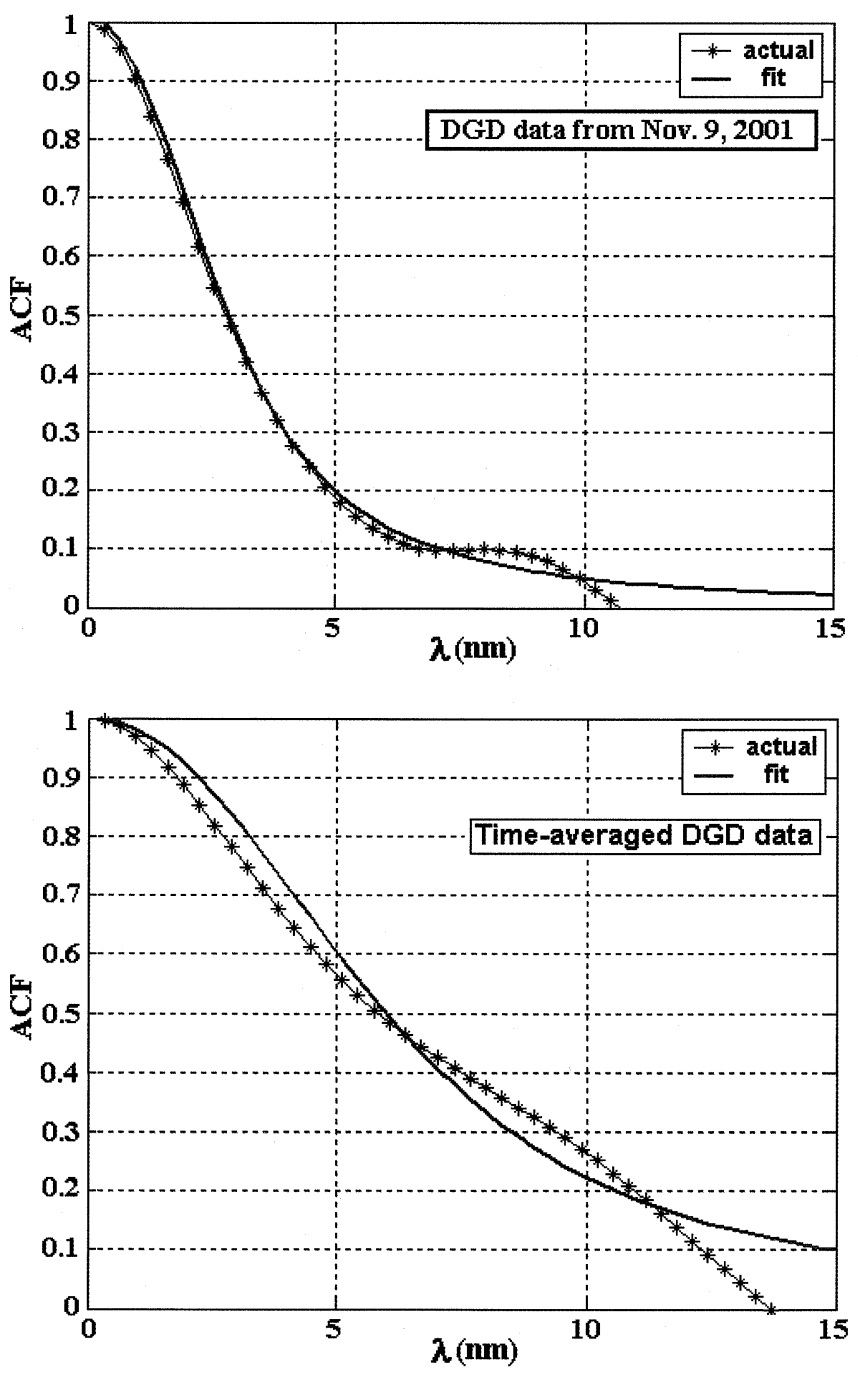

Fig. 6. Normalized spectral autocorrelation functions (ACFs) of normalized DGD data measured on November 9, 2001 (top) and time-averaged overall 692 measurements (bottom). Theoretical ACF curves are fitted to the measured spectral ACFs.

Thus, our observation of $3.4 \mathrm{~d}$ is consistent for the buried cable having no above-ground segments.

With knowledge gained from the temporal ACF analysis, we can now interpret realistically our DGD data set. Over the 86 d of observation, about 25 independent temporal samples per wavelength were collected.

\section{SPeCtral BehaVior of DGD}

From Fig. 1, we note that the DGD varies significantly with wavelength. In Fig. 5, the top plot shows the normalized spectral variation of the first DGD data (measured on November 9, 2001), and the bottom plot shows the spectral variation of the time-averaged, normalized DGD data, i.e., the normalized DGD data processed using (2).

To determine the DGD bandwidth, spectral autocorrelation analysis was performed on the normalized DGD spectral data. In Fig. 6, the top graph shows the resulting normalized spectral ACF for one spectral measurement (data collected on November 9,2001 ) and the bottom shows the normalized spectral ACF for 
the time-averaged data. Also shown in Fig. 6 are curves representing theoretical spectral ACFs for DGD, with the form [23]

$$
\operatorname{ACF}(\Delta \omega)=3 \frac{1-\exp \left(\frac{-\left\langle\Delta \tau^{2}\right\rangle \Delta \omega^{2}}{3}\right)}{\Delta \omega^{2}}
$$

where $\Delta \omega$ is the radian frequency and $\left\langle\Delta \tau^{2}\right\rangle$ represents the variance of the DGD.

From the measured data, the bandwidth for the normalized DGD is estimated to be about $7.5 \mathrm{~nm}$, or $936 \mathrm{GHz}$. Therefore, if the mean DGD is 1 ps and an optical channel is affected by significant DGD, nearby channels (within about $7.5 \mathrm{~nm}$ ) may also experience this effect.

Theory and experiments [23] have demonstrated that the DGD bandwidth is inversely proportional to the mean DGD, as follows:

$$
\omega_{c}=\frac{4 \sqrt{2}}{\langle\Delta \tau\rangle} .
$$

Thus, fibers with a high mean DGD have a narrower DGD bandwidth than fibers with a low mean DGD. Thus, for a fiber with a mean DGD of $1 \mathrm{ps}$, the predicted DGD bandwidth is $900 \mathrm{GHz}$, which agrees well with the bandwidth found using the spectral ACF fit in Fig. 6 (bottom). Note that the normalized DGD bandwidth in Fig. 6 (top) is about $4 \mathrm{~nm}$, which is significantly less than the approximately 7.5-nm bandwidth seen in Fig. 6 (bottom). This should not be interpreted to mean that the DGD bandwidth is varying; rather, the bandwidth estimate obtained using all of the data will be more accurate, since it is based on significantly more data points.

\section{IMPLICATIONS FOR NETWORK AVAILABILITY}

\section{A. Mean Time Between PMD-Related Outages}

In the past, the outage probability $P_{\text {out }}$ due to PMD effects has been expressed in terms of minutes per year [2]. In cases where the drift time is measured in days and the probability of an outage is quite small, $P_{\text {out }}$ represents the annualized outage probability based on long time records. Accurately estimating the impact of the PMD on network availability requires statistical analysis of the DGD variability. Caponi et al. [24] showed how the mean time between PMD-related outages can be estimated from the temporal characteristics of DGD variations and the Maxwellian pdf. The mean outage rate $R_{\text {out }}$ (defined as the mean number of outage events per unit time with units of events per year) is found using [24]

$$
\left.R_{\text {out }}=\frac{1}{2} f_{\tau} \text { (threshold }\right) \int_{-\infty}^{\infty} f_{\tau^{\prime}}\left(\Delta \tau^{\prime}\right)\left|\Delta \tau^{\prime}\right| d \Delta \tau^{\prime}
$$

where $f_{\tau}(\cdot)$ is the DGD pdf, $\Delta \tau^{\prime}$ is the time derivative of the DGD, and $f_{\tau^{\prime}}(\cdot)$ is the pdf of $\Delta \tau^{\prime}$. In this analysis, it is assumed that an outage results when the DGD value exceeds the threshold value. Caponi et al. [24] observed $\Delta \tau$ and $\Delta \tau^{\prime}$ to be statistically independent and also found that $R_{\text {out }}$ is cable- and installationdependent.

Fig. 7 shows the calculated outage probability $P_{\text {out }}$ and the mean outage rate $R_{\text {out }}$ for a given system threshold relative to

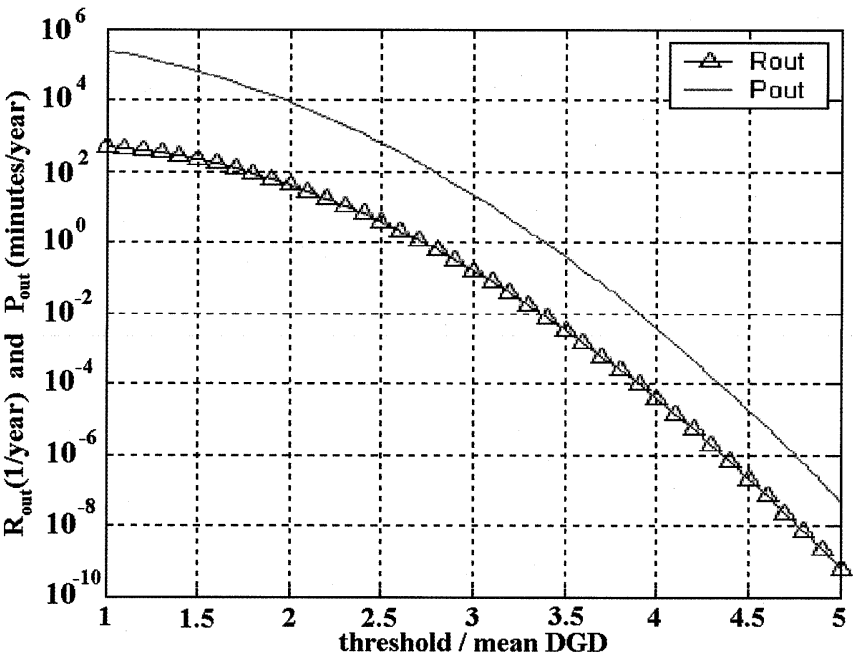

Fig. 7. Calculated outage probability $P_{\text {out }}$ and mean outage rate $R_{\text {out }}$ versus threshold/mean DGD.

the mean DGD. While $P_{\text {out }}$ is based only on the Maxwellian distribution, $R_{\text {out }}$ is based on measured DGD data. From our measured DGD data, we calculated an $R_{\text {out }}$ of 0.157 outage events per year (one outage event every $6.39 \mathrm{y}$ ) for the case where the threshold is three times the mean. When the threshold is increased to 3.7 times the mean DGD, $R_{\text {out }}$ becomes 0.0034 outage events per year, or one outage event in 1648 years.

For comparison, Nagel et al. [22] observed a DGD correlation time of $19 \mathrm{~h}$ and predicted that the DGD will exceed three times its mean value once every $3.5 \mathrm{y}$. From data measured on $37 \mathrm{~km}$ of buried cable (with above-ground segments) having a mean DGD of $9.44 \mathrm{ps,} \mathrm{Caponi} \mathrm{et} \mathrm{al.} \mathrm{[24]} \mathrm{predicted} \mathrm{that} \mathrm{the} \mathrm{DGD} \mathrm{will}$ exceed three times the mean DGD once every $2.5 \mathrm{y}$.

\section{B. Duration of High-DGD Events}

The mean duration of DGD-induced outages can be determined using statistical analysis, as well. Caponi et al. [24] showed that the mean outage duration $T_{\text {out }}$ is

$$
T_{\text {out }}=\frac{P_{\text {out }}}{R_{\text {out }}}
$$

which has units of minutes.

Fig. 8 shows the calculated mean outage duration $T_{\text {out }}$ as a function of system threshold relative to the mean DGD. Since $T_{\text {out }}$ is found using $R_{\text {out }}$, which is cable- and installation-dependent, $T_{\text {out }}$ will also be cable- and installation-dependent. For the case where the threshold is three times the mean DGD on this link, the mean duration of PMD-induced outages is about 136 $\min$. For the case where the threshold is increased to 3.7 times the mean DGD, $T_{\text {out }}$ reduces to about $108 \mathrm{~min}$.

Again for comparison, Nagel et al. [22] estimated a mean outage duration (outage means DGD greater than three times mean DGD) between 10 and 20 min for their link. Similarly, Caponi et al. [24] predicted a mean outage duration of $56 \mathrm{~min}$ on their cable. Furthermore, Bülow and Veith [15] found that while unusually long duration outages occur, the probability of occurrence decreases almost exponentially with outage duration. 


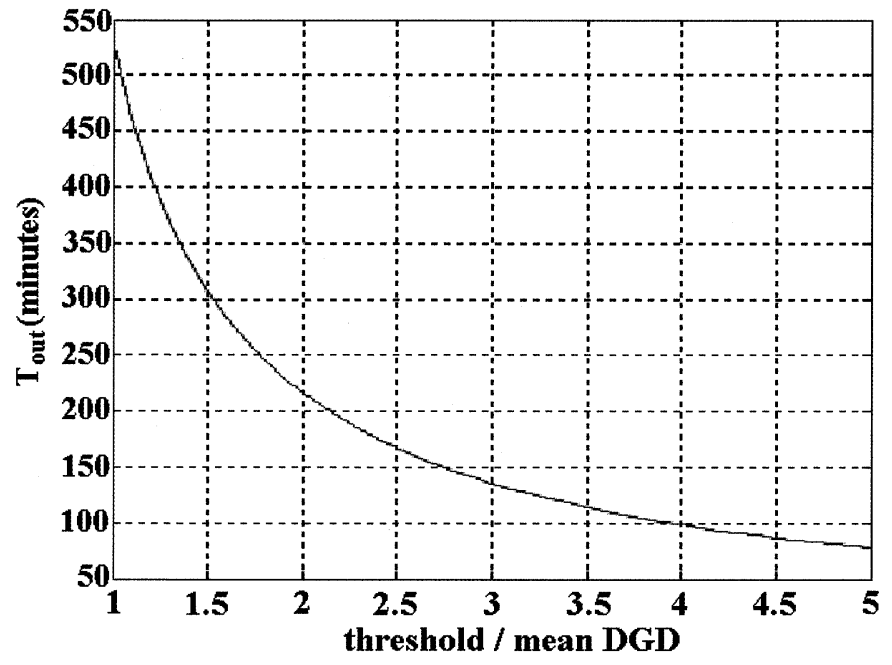

Fig. 8. Calculated mean outage duration $T_{\text {out }}$ as a function of threshold/mean DGD.

\section{Impact of High-DGD Events on Adjacent Channels}

When a high-DGD episode occurs, how many DWDM channels will be affected? For a link with a mean DGD of 5 ps, the DGD bandwidth will be about $180 \mathrm{GHz}$, or $1.44 \mathrm{~nm}$. Therefore, for a DWDM system with 50-GHz channel spacing, during a $3.7 \times\langle\Delta \tau\rangle$ event, the DGD in adjacent channels may also experience PMD-induced signal degradation (i.e., only two or three channels will likely be affected by a single high-DGD episode).

\section{Design Rules}

Based on these observations and analyses, certain rules may be developed. An important parameter in making decisions regarding PMD in a network is the ratio between the receiver's DGD tolerance $\Delta \tau_{\mathrm{RX}}$ and the link's mean DGD, as follows:

$$
M_{\tau}=\frac{\Delta \tau_{\mathrm{RX}}}{\langle\Delta \tau\rangle}
$$

For cases where $M_{\tau}>3$, the frequency of PMD-induced outages will be low, and their duration may be brief. In these cases, the approach proposed by Särkimukka et al. [13] (or one utilizing new protection techniques) may be viable. The occurrences that may require the switching of this traffic will likely be infrequent (spanning years) and may only be necessary for several minutes or a couple of hours.

For cases where $2<M_{\tau}<3$, PMD-induced outages may occur with a maximum frequency of one event every few days and a mean outage duration of 2-4 h. For cases where $M_{\tau}<2$, chronic PMD-induced outages will result with durations of several hours. In these instances, the option of applying PMD compensation, interrupting the link with a back-to-back terminal regenerator, or even replacing particular fiber segments, may be appropriate.

\section{E. Example Scenarios}

1) $10-\mathrm{Gb} / \mathrm{s},\langle\Delta \tau\rangle=10 \mathrm{ps}$, Receiver's DGD Tolerance 40 ps: In this scenario, the DGD margin $M_{\tau}$ is 4 . The probability of the DGD exceeding the receiver's DGD tolerance level is about $7.4 \times 10^{-9}$, or, effectively, 0 . In this case, it is quite unlikely that a PMD-induced outage will ever be observed, and if one does occur, its mean duration will be $100 \mathrm{~min}$. The DGD bandwidth will be about $90 \mathrm{GHz}$, or about $0.72 \mathrm{~nm}$.

2) $10-G b / s,\langle\Delta \tau\rangle=10 \mathrm{ps}$, Receiver's DGD Tolerance 23 ps: In this case, the margin $M_{\tau}$ will be 2.3 , meaning that the probability of the DGD exceeding the receiver's limit is about $0.37 \%$. For our buried cable, PMD-induced outages typically will occur about once a month and with a mean duration of about $3 \mathrm{~h}$. The DGD bandwidth will again be about $90 \mathrm{GHz}$.

3) 40-Gb/s, $\langle\Delta \tau\rangle=3.2 \mathrm{ps}$, Receiver's DGD Tolerance 5.7 ps: The DGD margin $M_{\tau}$, in this case, is 1.8 ; therefore, the probability of the DGD exceeding the receiver's limit is $4.4 \%$. In this scenario, PMD-induced outages typically will occur about every $6 \mathrm{~d}$. The mean duration will be about $4 \mathrm{~h}$; however, outages persisting for a day may occur. The DGD bandwidth is about $2.2 \mathrm{~nm}$, or $280 \mathrm{GHz}$, so in a DWDM application with $100-\mathrm{GHz}$ channel spacing, two or three channels may be affected during each outage.

\section{CONCLUSION}

By examining the statistical behavior of DGD in an optical fiber and using measured DGD data on a buried optical cable, predictions regarding the probability, frequency of occurrence, and spectral extent of high-DGD episodes can be made. Our observations indicate that DGD varies slowly in time and excursions of three or more times the mean DGD are infrequent and relatively short-lived. The measured DGD data indicate that for a PMDC system to be effective on this link, the PMDC system could have a time constant of a few hours and still keep pace with the DGD variations. Furthermore, since high-DGD events are isolated spectrally, a PMDC that is tunable in wavelength may be appropriate.

Viable mitigation approaches depend greatly on the DGD margin (i.e., the ratio of the receiver's maximum-tolerable DGD to the link's mean DGD). For cases where the link's mean DGD is comparable to the receiver's maximum-tolerable DGD, approaches for ensuring network availability include incorporation of PMDC systems, shortening the link length by strategically introducing back-to-back terminal regenerators or by replacing fiber segments found to have excessively high-DGD levels. For cases where the link's mean DGD is less than a third of the receiver's tolerable DGD, network reliability may be enhanced by providing a few spare channels in a DWDM environment. This finding is significant for network operators who may consider an optical networking solution whereby traffic may efficiently share protection bandwidth rather than extensive use of PMDC systems.

\section{ACKNOWLEDGMENT}

The authors thank F. Yarkosky for his leadership and support.

\section{REFERENCES}

[1] E. Iannone, F. Matera, A. Mecozzi, and M. Settembre, Nonlinear Optical Communication Networks. New York: Wiley, 1998, pp. 30-35.

[2] C. D. Poole and J. Nagel, "Polarization effects in lightwave systems," in Optical Fiber Telecommunications, I. P. Kaminow and T. L. Koch, Eds. San Diego, CA: Academic, 1997, vol. III A. 
[3] S. Betti, F. Curti, B. Daino, G. De Marchis, E. Iannone, and F. Matera, "Evolution of the bandwidth of the principal states of polarization in single-mode fibers," Opt. Lett., vol. 16, no. 7, pp. 467-469, 1991.

[4] F. Curti, B. Daino, G. de Marchis, and F. Matera, "Statistical treatment of the evolution of the principal states of polarization in single-mode fibers," J. Lightwave Technol., vol. 8, pp. 1162-1166, Aug. 1990.

[5] N. Gisin, R. Passy, J. C. Bishoff, and B. Perry, "Experimental investigation of the statistical properties of polarization-mode dispersion in single mode fibers," IEEE Photon. Technol. Lett., vol. 5, pp. 819-821, July 1993.

[6] E. Iannone, F. Matera, A. Galtarossa, G. Gianello, and M. Schiano, "Effect of polarization dispersion on the performance in IM-DD communication systems," IEEE Photon. Technol. Lett., vol. 5, pp. 1247-1249, Oct. 1993.

[7] R. Khosravani and A. E. Willner, "Comparison of different modulation formats in terrestrial systems with high polarization mode dispersion," in Proc. OFC2000, Baltimore, MD, 2000, WL5, pp. 201-203.

[8] W. Shieh, H. Haunstein, B. Mckay, D. Fishman, A. Golubchik, J. Diubaldi, C. Martell, V. Arya, R. Lee, and H. Choudhury, "Dynamic polarization-mode-dispersion compensation in WDM systems," in Proc. ECOC2000, vol. II(4.2.5), Munich, Germany, 2000, pp. 41-43.

[9] Y. Xie, Q. Yu, L.-S. Yan, O. H. Adamczyk, Z. Pan, S. Lee, A. E. Willner, and C. R. Menyuk, "Enhanced PMD mitigation using forward-error-correction coding and a first-order compensator," in Proc. OFC2001, Anaheim, CA, 2001, WAA2.

[10] H. Rosenfeldt, Ch. Knothe, R. Ulrich, E. Brinkmeyer, U. Feiste, C. Schubert, J. Berger, R. Ludwig, H. G. Weber, and A. Ehrhardt, "Automatic PMD compensation at $40 \mathrm{Gbit} / \mathrm{s}$ and $80 \mathrm{Gbit} / \mathrm{s}$ using a three-dimensional DOP evaluation for feedback," in Proc. OFC2001, Anaheim, CA, 2001, PD27.

[11] N. Kikuchi, "Analysis of signal degree of polarization degradation used as control signal for optical polarization mode dispersion compensation," J. Lightwave Technol., vol. 19, pp. 480-486, Apr. 2001.

[12] H. Y. Pua, K. Peddanarappagari, B. Zhu, C. Allen, K. Demarest, and R. Hui, "An adaptive first-order polarization-mode dispersion compensation system aided by polarization scrambling: Theory and demonstration," J. Lightwave Technol., vol. 18, pp. 832-841, June 2000.

[13] S. Särkimukka, A. Djupsjöbacka, A. Gavler, and G. Jacobsen, "Mitigation of polarization-mode dispersion in optical multichannel systems," J. Lightwave Technol., vol. 18, pp. 1374-1380, Oct. 2000.

[14] M. Karlsson, J. Brentel, and P. A. Andrekson, "Long-term measurement of PMD and polarization drift in installed fibers," J. Lightwave Technol., vol. 18, pp. 941-951, July 2000.

[15] H. Bülow and G. Veith, "Temporal dynamics of error-rate degradation induced by polarization mode dispersion of an installed field fiber link," in Proc. ECOC1997, vol. 1, Edinburgh, Scotland, 1997, Mo3C, pp. $115-118$.

[16] C. D. Poole, R. W. Tkach, A. R. Chaplyvy, and D. A. Fishman, "Fading in lightwave systems due to polarization-mode dispersion," IEEE Photon. Technol. Lett., vol. 3, pp. 68-70, Jan. 1991.

[17] S. Bahsoun, J. Nagel, and C. Poole, "Measurements of temporal variations in fiber transfer characteristics to $20 \mathrm{GHz}$ due to polarization-mode dispersion," in Proc. ECOC1990, Amsterdam, 1990, Postdeadline Paper, pp. 1003-1006.

[18] J. Cameron, L. Chen, X. Bao, and J. Stears, "Time evolution of polarization mode dispersion in optical fibers," IEEE Photon. Technol. Lett., vol. 10, pp. 1265-1267, Sept. 1998.

[19] T. Takahashi, T. Imai, and M. Aiki, "Time evolution of polarization mode dispersion in $120 \mathrm{~km}$ installed optical submarine cable," Electron. Lett., vol. 29, no. 18, pp. 1605-1606, 1993.

[20] T. Kawazawa and Y. Namihira, "Long-term polarization-mode-dispersion measurement of installed optical submarine cable," in Proc. OFC1994, 1994, pp. 228-229.

[21] C. De Angelis, A. Galratossa, G. Gianello, F. Marera, and M. Schiano, "Time evolution of polarization-mode dispersion in long terrestrial links," J. Lightwave Technol., vol. 10, pp. 552-555, May 1992.

[22] J. A. Nagel, M. W. Chbat, L. D. Garrett, J. P. Soigné, N. A. Weaver, B. M. Desthieux, H. Bülow, A. R. McCormick, and R. M. Derosier, "Long-term PMD mitigation at $10 \mathrm{~Gb} / \mathrm{s}$ and time dynamics over high-PMD installed fiber," in Proc. ECOC2000, vol. II(4.2.1), Munich, 2000, pp. 31-32.

[23] M. Karlsson and J. Brentel, "Autocorrelation function of the polarization-mode dispersion vector," Opt. Lett., vol. 24, no. 14, pp. 939-941, 1999 .
[24] R. Caponi, B. Riposati, A. Rossaro, and M. Schiano, "WDM design issues with highly correlated PMD spectra of buried optical fibers," in Proc. OFC2002, Anaheim, CA, 2002, ThI5, pp. 453-455.

Christopher T. Allen (M'94-SM'95) was born in Independence, MO, on October 7, 1958. He received the B.S., M.S., and Ph.D. degrees in electrical engineering from the University of Kansas, Lawrence, in 1980, 1982, and 1984, respectively.

From 1984 to 1990, he was with Sandia National Laboratories in Albuquerque, NM, working in exploratory radar systems and development of high-speed digital systems. From 1990 to 1994, he was with the Allied Signal Kansas City Division, Kansas City, MO, where he worked in the areas of high-speed digital design, radar systems analysis, and multichip module development. Since August 1994, he has been a Faculty Member in the Electrical Engineering and Computer Science Department at the University of Kansas. Currently, he is the Director of the Radar Systems and Remote Sensing Laboratory and Co-Director of the Lightwave Communication Systems Laboratory. He has been a Technical Reviewer for Remote Sensing of the Environment, Geophysics-The Journal of the Society of Exploration Geophysicists, and Journal of Glaciology. His research interests include high-speed digital circuits, microwave remote sensing, radar systems, and photonics/lightwave technologies.

Dr. Allen has served as a Technical Reviewer for various IEEE journals. He also serves on the SAE AE-8D task group on standards development for fiberoptic cable and test methods for aerospace applications. He is a Member of Phi Kappa Phi, Tau Beta Pi, Eta Kappa Nu, and the International Union of Radio Science (URSI).

Pradeep Kumar Kondamuri was born in Nellore, India, on August 11, 1978. $\mathrm{He}$ received the B.tech. degree in electronics and communications engineering from Sri Venkateswara University, Tirupathi, India, in 2000, and the M.S. degree in electrical engineering from the University of Kansas, Lawrence, in 2002 . He is currently working toward the Ph.D. degree in electrical engineering with the University of Kansas.

His research interests include optical fiber communications, digital signal processing for telecommunications, and microwave remote sensing.

Douglas L. Richards received the B.S. and M.S. degrees in electrical engineering from the University of Missouri-Rolla.

He joined Sprint in 1998. He had worked in Optical Engineering and Standards before transferring into the Technology, Planning, and Integration (TP\&I) Department. Currently, he is a Senior Member of the Technical Staff within TP\&I. He works at the physical transport layers within a Long-Term Technology Planning group that coordinates Sprint's technology direction statements. Part of his responsibility is to co-manage TP\&I's research in this area (i.e., lightwave projects at $\mathrm{KU}$ - exploring fiber compatibility issues and technologies that would improve long-haul transmission efficiencies). He is also active in ITU-T SG-15/Q.16\&17.

Douglas C. Hague received the B.S. degree in engineering physics from the University of Tulsa, Tulsa, OK, in 1989, and the M.S. degree in metals science and engineering and the Ph.D. degree in materials science and engineering from Pennsylvania State University (Penn State) in 1992 and 1995, respectively. He also received the M.S. degree in system design and management from the Massachusetts Institute of Technology, Cambridge, in 2001.

At Penn State, he worked to develop processes and simulations for the production and consolidation of nanocrystalline ceramic powers. From 1995 through 2000, he held various positions in the research, design, and production of thermal barrier coatings and jet engines at Pratt \& Whitney in Florida and Connecticut. Since 2001, he has been working in the Technology, Planning, and Integration Department within Sprint Corporation in Overland Park, KS, where his research interests include polarization-mode dispersion. 